
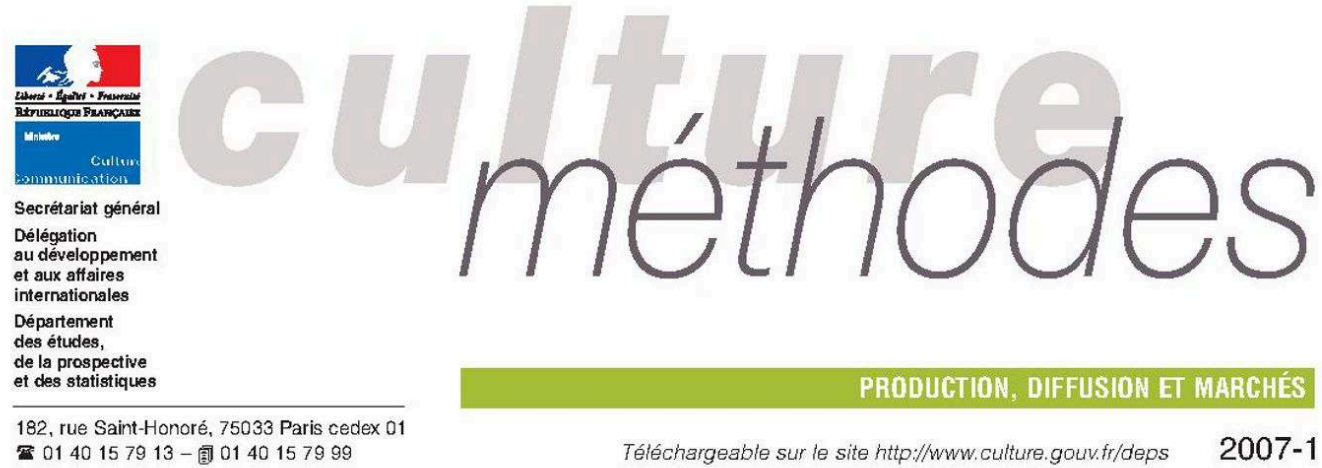

\title{
Les premiers principes de l'analyse d'impact économique local d'une activité culturelle
}

\section{Yann Nicolas*}

\section{Avant-propos}

Le DEPS inaugure une nouvelle collection, «Culture méthodes». Elle met à disposition du public des notes de méthode dans les domaines qui relèvent des compétences du Département des études, de la prospective et des statistiques : statistique, analyse économique et sociologique dans les champs du Ministere de la culture et de la communication. Cette collection s'adresse aux professionnels de la culture, aux services administratifs culturels, aux élus, aux évaluateurs, comme aux milieux de la recherche. À partir d'une investigation de la lit térature théorique et empirique, l'auteur précise les premiers principes de l'analyse d'impact économique local d'une activité culturelle. Au-delà de l'intérêt de faire connâtre ces principes avec un souci d'exactitude et de rigueur, cette note doit permettre de favoriser des approches comparables en répondant au renouvellement d'intérêt pour l'analyse économique manifesté par de nombreux acteurs de la culture.

$$
\text { P. C. }
$$

Cette note de méthode rappelle les premiers principes de l'analyse d'impact économique local d'un équipement ou d'un événement culturel. Ces principes portent uniquement sur l'analyse d'impact traditionnelle ou conventionnelle, celle qui cherche à estimer, pour une zone enviromnante donnée, l'ampleur des revenus et emplois locaux additionnels crés à court terme grâce aux dépenses des visiteurs ou spectateurs directement attribuables à l'activité culturelle étudiée.

Ce rappel paraît nécessaire pour au moins deux raisons. La première est la diversité des approches retenues dans les études d'impact économique rendues publiques et celle des conclusions qui ont pu en être tirées. La seconde est le regain d'intérêt actuel pour des résultats d'analyse d'impact économique chez différents acteurs de la culture, qu'il s'agisse de responsables d'équipement ou d'organisateurs d'événement, de directions ministérielles ou régionales culturelles, de collectivités locales ou d' «évaluateurs».

La définition de l'analyse d'impact économique est préalable à l'exposé de ses premières règles générales et celui de ses limites, lorsque ses résultats sont invoqués pour motiver le soutien public à des activités culturelles'.

\section{ÉLÉMENTS DE DÉFINITION}

L'analyse d'impact économique est la méthode retenue par toute étude dite d'impact économique. Qu'est-ce qu'une étude d'impact économique ? Que mesure-t-elle exactement ? Fondée sur un circuit économique sous-jacent et un processus multiplicateur, ce type d'étude résulte d'une analyse par nature locale.

\section{Le raisonnement fondamental}

Il s'agit de mesurer l'impact de $x$ pour une certaine zone :

- $x$ peut être un équipement culturel (monument, site patrimonial, musée...), un événement culturel (festival, exposition temporaire, spectacle de rue...), un ensemble d'équipements culturels, une série d'événements culturels ou un secteur culturel entier;

\footnotetext{
* Économiste chargé d'études au DePs. Chargé de cours à l'université Paris VII-Denis Diderot et Paris XII-Val-de-Marne.

1. Cette note s'inspire directement de plusieurs articles et ouvrages. Une première liste de reférences se trouve à la page 18 du document de travail suivant : Yann Nicolas, L'analyse d'impact économique de la culture. Principes et limites, document de travail $\mathrm{n}^{\circ} 1271$, Paris, ministère de la Culture et de la Communication, Délégation au développement et à l'action internationale, Deps, juillet 2006.
} 


\section{Les Premiers Principes de l'analyse d'impact économique local d'une activité culturelle}

How to evaluate the local economic impact of a cultural event : first methodological rules

\section{Yann Nicolas}

Éditeur : Département des études, de la prospective et des statistiques

Lieu d'édition : Paris

Année d'édition : 2007

Date de mise en ligne : 21 septembre 2015

Collection : Culture méthodes

ISBN électronique : 9782111398672

\section{Sobooks}

http://books.openedition.org

\section{Édition imprimée}

Date de publication : 30 avril 2007

Nombre de pages : 8

\section{Référence électronique}

NICOLAS, Yann. Les Premiers Principes de l'analyse d'impact économique local d'une activité culturelle. Nouvelle édition [en ligne]. Paris : Département des études, de la prospective et des statistiques, 2007 (généré le 25 avril 2021). Disponible sur Internet : <http://books.openedition.org/deps/324>. ISBN : 9782111398672 

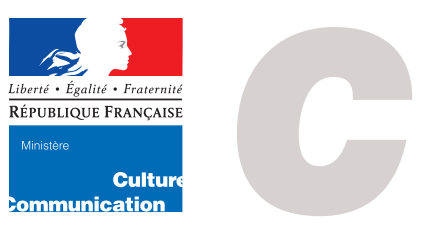

Secrétariat général Délégation

au développement

et aux affaires

internationales

Département

des études,

de la prospective

et des statistiques

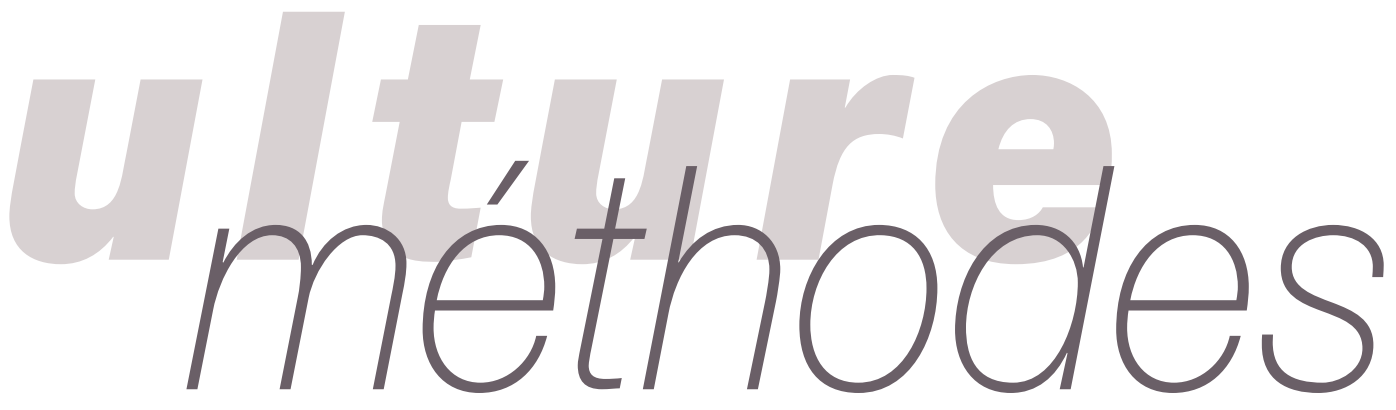

182, rue Saint-Honoré, 75033 Paris cedex 01

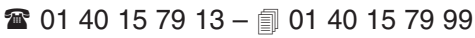

\title{
Les premiers principes de l'analyse d'impact économique local d'une activité culturelle
}

\author{
Yann Nicolas*
}

\section{Avant-propos}

Le DEPS inaugure une nouvelle collection, «Culture méthodes». Elle met à disposition du public des notes de méthode dans les domaines qui relèvent des compétences du Département des études, de la prospective et des statistiques : statistique, analyse économique et sociologique dans les champs du Ministère de la culture et de la communication. Cette collection s'adresse aux professionnels de la culture, aux services administratifs culturels, aux élus, aux évaluateurs, comme aux milieux de la recherche. À partir d'une investigation de la littérature théorique et empirique, l'auteur précise les premiers principes de l'analyse d'impact économique local d'une activité culturelle. Au-delà de l'intérêt de faire connaître ces principes avec un souci d'exactitude et de rigueur, cette note doit permettre de favoriser des approches comparables en répondant au renouvellement d'intérêt pour l'analyse économique manifesté par de nombreux acteurs de la culture.

P. C.
Cette note de méthode rappelle les premiers principes de l'analyse d'impact économique local d'un équipement ou d'un événement culturel. Ces principes portent uniquement sur l'analyse d'impact traditionnelle ou conventionnelle, celle qui cherche à estimer, pour une zone environnante donnée, l'ampleur des revenus et emplois locaux additionnels créés à court terme grâce aux dépenses des visiteurs ou spectateurs directement attribuables à l'activité culturelle étudiée.

Ce rappel paraît nécessaire pour au moins deux raisons. La première est la diversité des approches retenues dans les études d'impact économique rendues publiques et celle des conclusions qui ont pu en être tirées. La seconde est le regain d'intérêt actuel pour des résultats d'analyse d'impact économique chez différents acteurs de la culture, qu'il s'agisse de responsables d'équipement ou d'organisateurs d'événement, de directions ministérielles ou régionales culturelles, de collectivités locales ou d'«évaluateurs ».

La définition de l'analyse d'impact économique est préalable à l'exposé de ses premières règles générales et celui de ses limites, lorsque ses résultats sont invoqués pour motiver le soutien public à des activités culturelles ${ }^{1}$.

\section{ÉLÉMENTS DE DÉFINITION}

L'analyse d'impact économique est la méthode retenue par toute étude dite d'impact économique. Qu'est-ce qu'une étude d'impact économique ? Que mesure-t-elle exactement? Fondée sur un circuit économique sous-jacent et un processus multiplicateur, ce type d'étude résulte d'une analyse par nature locale.

\section{Le raisonnement fondamental}

Il s'agit de mesurer l'impact de x pour une certaine zone :

- x peut être un équipement culturel (monument, site patrimonial, musée...), un événement culturel (festival, exposition temporaire, spectacle de rue...), un ensemble d'équipements culturels, une série d'événements culturels ou un secteur culturel entier ;

\footnotetext{
* Économiste chargé d'études au DEPS. Chargé de cours à l'université Paris VII-Denis Diderot et Paris XII-Val-de-Marne.

1. Cette note s'inspire directement de plusieurs articles et ouvrages. Une première liste de références se trouve à la page 18 du document de travail suivant : Yann Nicolas, L'analyse d'impact économique de la culture. Principes et limites, document de travail $\mathrm{n}^{\circ} 1271$, Paris, ministère de la Culture et de la Communication, Délégation au développement et à l'action internationale, Deps, juillet 2006.
} 
- la zone peut être une ville, une agglomération, un canton, un département, une région, un pays, etc.

Le graphique 1 représente le circuit économique fondamental sous-jacent de toute étude d'impact. Il montre :

- que les résidents locaux et les visiteurs ou spectateurs non locaux paient initialement des impôts et des taxes aux autorités publiques de la zone ;

- que ces autorités locales utilisent une partie de leurs recettes fiscales pour subventionner $\mathrm{x}$ sur fonds publics ;

- que $\mathrm{x}$ attire des visiteurs ou spectateurs non locaux qui dépensent dans la zone ;

- que ces dépenses nouvelles extérieures créent de nouveaux revenus et des emplois dans la zone au profit des résidents locaux, ce qui complète le cycle vertueux de développement économique local.

D'un certain point de vue, les résidents locaux, aidés par les taxes payées par les visiteurs ou spectacteurs non locaux, ont pour fonction de fournir les fonds initiaux et reçoivent un retour sur investissement sous la forme d'emplois nouveaux et de plus de revenus.

Une étude d'impact économique a pour objectif d'estimer l'ampleur des revenus et emplois locaux supplémentaires ainsi créés.

Une étude d'impact cherche donc à répondre à la question suivante : de combien l'activité économique de court terme déclinerait dans une zone considérée si x n'existait plus dans cette zone ? Ou, dans le cas d'un projet culturel, de combien cette activité croîtrait dans la zone si x existait? Une étude d'impact s'intéresse précisément à la hausse nette de l'activité économique due aux injections externes ou nettes de nouvelles dépenses dans la zone comme résultat direct de l'existence de $\mathrm{x}$. Les injections externes de nouvelles dépenses sont celles des visiteurs ou spectateurs non locaux qu'on peut attribuer au motif premier de visite ou de présence qu'est x, c'est-à-dire les injections nettes des injections locales. Visiteurs ou spectateurs non locaux doivent être compris au sens large ; ils peu- vent ainsi inclure, de l'extérieur de la zone, des visiteurs ou spectateurs privés mais aussi des artistes, des techniciens artistiques, des journalistes, des vendeurs, des exposants, des bénévoles, des commanditaires (mécènes), des investisseurs, etc.

\section{Le processus du multiplicateur}

L'analyse d'impact repose sur le concept économique de «multiplicateur». Lorsque des visiteurs ou des spectateurs non locaux dépensent en billetterie, restauration, hébergement et commerce de détail dans la zone d'étude du fait de l'existence de $\mathrm{x}$, leurs dépenses directes initiales stimulent l'activité économique locale et créent un supplément de chiffres d'affaires, de rémunérations, d'emplois et de recettes fiscales. L'hypothèse implicite à ce raisonnement est que les secteurs d'activité locaux sont interdépendants. Une injection monétaire non locale peut ainsi créer une suite de répercussions dans l'ensemble des secteurs, notamment non culturels. L'ampleur de ces répercussions dépend d'un certain nombre de fuites dans le circuit et seuls les euros restant à l'intérieur de la zone, après fuites, constituent un gain pour l'économie locale.

Le graphique 2 représente le processus du multiplicateur en explicitant ces répercussions et ces fuites.

Il y est supposé que les visiteurs ou spectateurs non locaux dépensent dans quatre types d'établissement de la zone d'étude. Cette injection initiale de dépenses constitue l'impact économique dit direct. Ces fonds initiaux reçus par ces établissements locaux peuvent alors être injectés dans la zone de trois façons :

a) avec les autres entreprises locales pour réapprovisionner leurs stocks pour les ventes futures, pour entretenir les bâtiments, installations et équipements, pour payer les primes d'assurance, et bien d'autres objets (achats locaux entre branches) ;

b) avec les salariés et actionnaires locaux sous la forme de salaires, de rémunérations ou de dividendes qui constituent

Graphique 1 - Le circuit économique sous-jacent

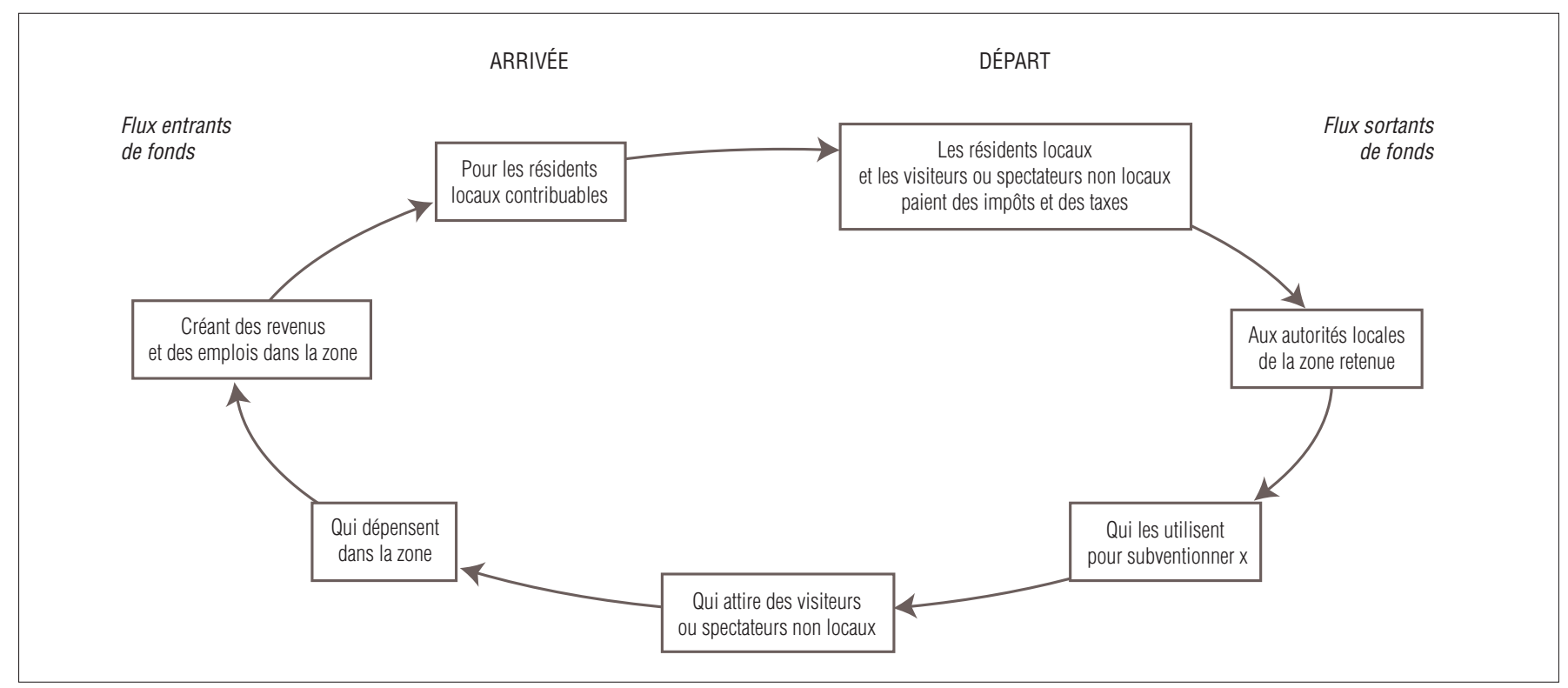

Source : Culture méthodes (DEPS, Ministère de la culture, 2007), d'après Crompton ${ }^{2}$

2. John L. CROMPton, “Economic Impact Studies: Instruments for Political Shenanigans?”, Journal of Travel Research, vol. 45, nº 1, août 2006, p. 67-82. 


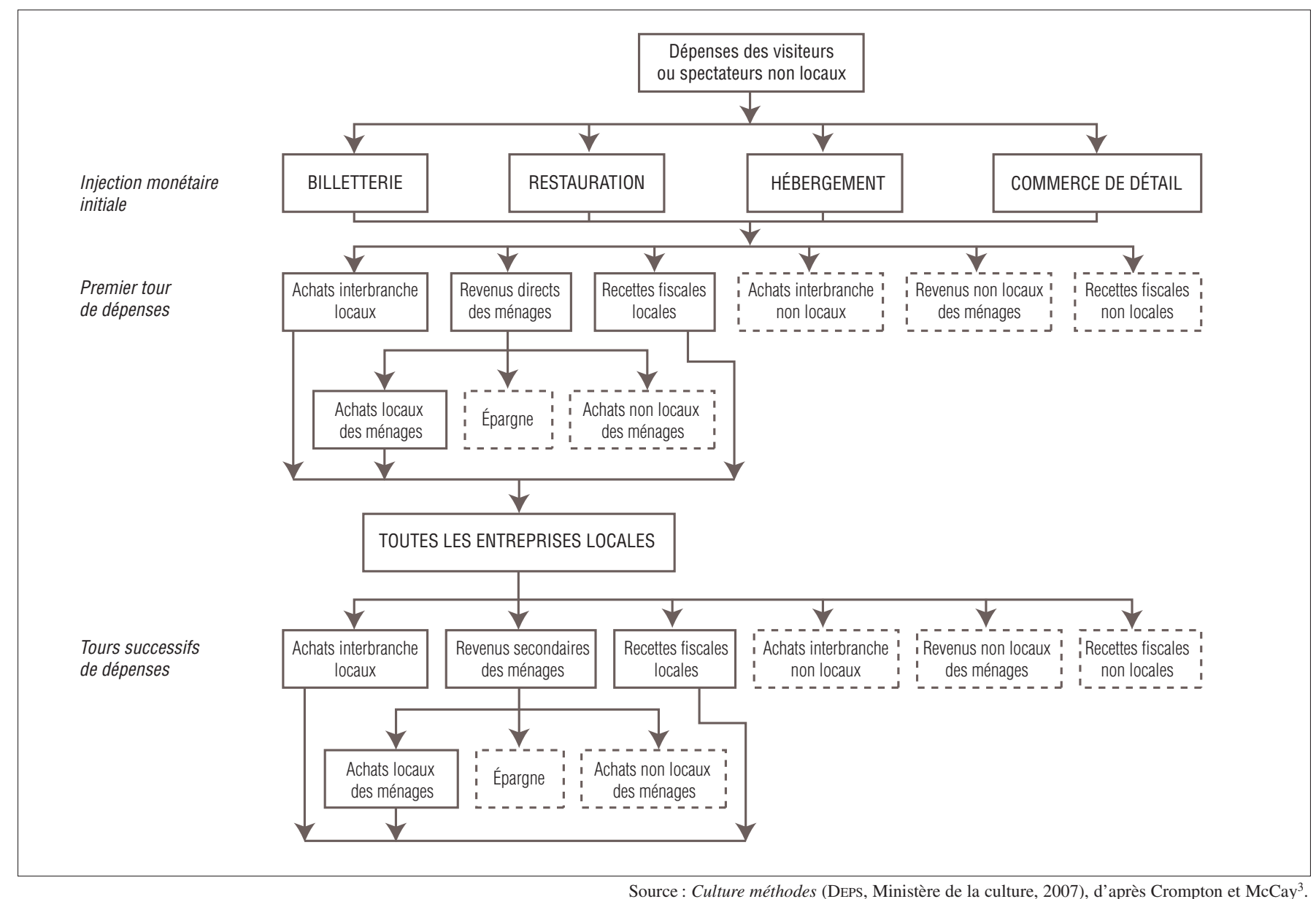

des revenus individuels pour eux (revenus directs des ménages);

c) avec les autorités locales sous forme d'impôts locaux tels que, par exemple, les taxes foncières, d'habitation, professionnelle, de séjour, etc. (recettes fiscales locales).

Ces fonds initiaux reçus par ces établissements locaux peuvent aussi être dépensés hors de la zone de trois façons :

a) avec les entreprises non locales hors de la zone (achats entre branches non locaux);

b) avec les salariés et actionnaires non locaux sous la forme de salaires, de rémunérations ou de dividendes qui constituent des revenus individuels pour eux (revenus non locaux des ménages);

c) avec les autorités non locales sous forme d'impôts tels que, par exemple, l'impôt sur les sociétés, la taxe sur la valeur ajoutée, etc. (recettes fiscales non locales).

Ces trois dernières catégories de dépenses correspondent à des fuites (cases en pointillé sur le graphique 2), c'est-à-dire des sommes qui ne restent pas à l'intérieur de la zone et constituent ainsi une perte pour l'économie locale 4 . En outre, une

3. John L. CROMPton et Stacey L. MCCAY, "Measuring the Economic Impact of Festivals and Events: Some Myths, Misapplications and Ethical Dilemmas", Festival Management and Event Tourism, vol. 2, $\mathrm{n}^{\circ} 1,1994$, p. 33-43.

4. Il est possible que des salariés non locaux dépensent une partie de leur budget à l'intérieur de la zone. Cette possibilité n'est cependant pas indiquée sur le graphique 2 car il est probable que ces dépenses sont relativement faibles dans de nombreux cas et le fait de les inclure compliquerait peu utilement la figure. partie des revenus directs reçus par les résidents locaux peut ne pas être dépensée dans l'économie locale mais plutôt épargnée ou dépensée à l'extérieur de la zone. Dans l'approche de court terme qui est celle de l'analyse d'impact traditionnelle ou conventionnelle, il s'agit là aussi de fuites.

Comme le montre le graphique 2 , les dépenses initiales des visiteurs ou spectateurs se diffusent ensuite progressivement par tours successifs dans l'économie locale en suivant le même processus, avec une part de fuites hors de la zone à chaque tour, jusqu'à ce que ces dépenses atteignent une ampleur négligeable. Ces tours successifs d'activité économique reflétant les dépenses issues d'achats entre branches locaux et des recettes fiscales locales sont appelés «impacts indirects ». La proportion du revenu des ménages locaux qui est dépensée localement en biens et services est appelée « impact induit »; il s'agit de la hausse de l'activité économique locale produite par l'augmentation de la consommation locale due à celle des différents types de revenus des résidents locaux.

Au total, les effets directs sont les effets de premier tour des dépenses des visiteurs non locaux en billetterie, restauration, hébergement et commerce de détail (dépenses des établissements concernés); les effets indirects correspondent à la répercussion des tours additionnels de re-circulation des sommes initiales par le biais des dépenses des entreprises locales et des autorités locales ; les effets induits sont les répercussions produites ultérieurement par les effets directs et indirects et causées par les salariés d'entreprises locales bénéficiaires dépensant une partie de leurs rémunérations dans d'autres entreprises locales. 
Une étude d'impact économique a pour objectif d'estimer l'importance de ces trois types d'effets à l'aide de trois critères: les ventes additionnelles au profit des entreprises locales, les revenus individuels locaux (ménages) et les emplois locaux supplémentaires créés 5 .

\section{Une analyse par nature locale}

L'analyse d'impact n'a de sens qu'à un niveau local. En effet, au niveau national, ce que gagne une région en réussissant à attirer des dépenses non locales grâce à $\mathrm{x}$ est perdu pour les autres régions nationales, une dépense décidée ici étant compensée par une économie ailleurs. Ce que gagne par exemple la région Provence-Alpes-Côte d'Azur grâce à ses activités patrimoniales culturelles en réussissant à attirer des visiteurs bretons est perdu pour la région Bretagne, et inversement. Au plan national, le revenu national n'a pas augmenté grâce à $x$ mais est seulement partagé différemment.

L'importance de ce gain pour une région peut être nuancée d'une autre façon en remarquant que les régions perdantes sont sans doute des partenaires commerciaux de la région gagnante. $\mathrm{Si}$, certes, elles perdent les sommes attirées par l'existence de $\mathrm{x}$, cette perte monétaire est aussi ressentie par la région gagnante. Inversement, les partenaires commerciaux de cette dernière, parmi lesquels les régions perdantes, bénéficient de sa plus grande richesse. Pertes et gains circulent ainsi de manière complexe et continûment. Par définition localisée par son objet, une étude d'impact est susceptible d'introduire un biais dans le sens d'une surestimation de l'effet économique net pour la région gagnante.

Quoi qu'il en soit, au niveau national, le jeu n'est pas à somme nulle uniquement grâce au gain - potentiellement très important - tiré du fait de réussir à attirer sur le sol national les dépenses de visiteurs étrangers, lorsque, précisément, l'économie est dite ouverte. Cependant, là encore, le gain doit être modéré en reprenant le raisonnement ci-dessus pour l'appliquer cette fois à des pays plutôt qu'à des régions.

Enfin, au niveau international, compte tenu de l'approche de court terme de l'analyse d'impact économique traditionnelle ou conventionnelle, le jeu est nécessairement à somme nulle.

\section{Premières RèGLes GÉNÉRALES DE MÉTHODE}

Effets directs, indirects et induits doivent être estimés en respectant plusieurs règles économiques de calcul, en excluant plusieurs types de dépenses et en cherchant à tenir compte des coûts qui peuvent être directement associés à l'objet d'étude.

5. Signalons qu'il existe dans la littérature d'autres définitions de ces trois types d'effets.

\section{Utiliser un modèle économique local ad hoc}

Seule l'utilisation d'un modèle « entrées/sorties » de l'économie locale choisie permet d'obtenir, sur une base empirique réelle, une estimation raisonnable des trois types d'effets décrits ${ }^{6}$.

Un modèle entrées/sorties est un système comptable élaboré qui suit la trace des transactions et des flux de toute nouvelle somme partout dans une économie. Il est constitué de tableaux dits entrées/sorties qui désagrègent l'économie étudiée en un certain nombre de secteurs et branches et examinent les flux monétaires de biens et services entre eux. Ce type de modèle permet de calculer des coefficients multiplicateurs (totaux) associés aux ventes, revenus et emplois locaux à l'aide de l'estimation de l'injection initiale de dépenses.

Cette estimation - nécessaire mais non suffisante ${ }^{7}$ - se fait à partir de données recueillies par enquête auprès des visiteurs ou auprès des billetteries, restaurateurs, «hébergeants » et détaillants locaux. Bien que les deux sources d'informations puissent être utilement croisées, la première est néanmoins préférable à la seconde pour au moins deux raisons : les entreprises locales rencontreront des difficultés à évaluer la part des dépenses de leurs clients qui provient de visiteurs non locaux plutôt que de résidents locaux; ces entreprises auront en outre des difficultés à savoir si l'existence de $\mathrm{x}$ a prioritairement influé ou pas sur la décision des visiteurs non locaux de venir dans la zone d'étude.

La formule générale des coefficients multiplicateurs est la suivante :

effets directs + effets indirects + effets induits

dépenses injectées des visiteurs non locaux

$\grave{A}$ titre d'exemple, un coefficient pour les revenus individuels de $0,65=0,36+0,08+0,21$, qui décompose les effets directs, indirects et induits d'un euro supplémentaire de dépenses des visiteurs non locaux dans l'économie locale, signifiera que pour tout euro extérieur injecté, 65 centimes de revenus individuels reviendront dans celle-ci sous la forme de salaires, de rémunérations ou de dividendes pour les résidents locaux.

L'estimation de ces coefficients multiplicateurs suppose le respect de trois principes supplémentaires:

- ne pas choisir intentionnellement la taille de la zone d'étude en sachant que celle-ci peut influer sur l'importance des coefficients estimés. Il est en effet probable qu'un coefficient pour une petite zone (une ville par exemple) sera plus faible qu'un coefficient pour une plus grande (une région par exemple) car, en règle générale, une zone plus petite tend à ne pas bénéficier d'interdépendances sectorielles suffisantes pour faciliter la rétention des sommes non locales dépensées durant le premier tour de dépenses ;

\footnotetext{
6. À notre connaissance, il semble qu'il n'existe pas à l'heure actuelle en France de modèles entrées/sorties locaux pour des régions, des départements, voire des grandes villes. Signalons en outre que l'utilisation alternative de modèles locaux «d'équilibre général calculable » a été récemment faite dans la littérature.

7. Pour la simple raison qu'une même injection initiale a peu de chances de produire un même impact pour deux économies locales différentes, chacune d'elles étant vraisemblablement différente structurellement (voir ci-après).
} 
- faire porter les coefficients multiplicateurs sur les revenus individuels locaux et sur les emplois locaux, et non sur les ventes des entreprises locales. En effet, au regard du graphique 1 , les coefficients multiplicateurs pour les revenus individuels et les emplois offrent des estimations qui ont des implications pratiques importantes pour les parties prenantes $^{8}$. À l'inverse des coefficients pour les ventes, les coefficients pour les revenus et pour les emplois permettent de lier les bénéfices économiques reçus par les résidents locaux aux recettes fiscales qu'ils ont investies. Une seconde raison, complémentaire et plus prosaïque, tient à ce que les estimations pour les ventes sont souvent plus importantes que celles pour les revenus individuels. Il peut alors être tentant de rapporter l'impact en termes de ventes plutôt qu'en termes de revenus individuels. Des chiffres plus élevés apparaîtraient ainsi mieux justifier l'investissement public local qu'il peut s'agir de défendre, bien qu'ils soient dénués de sens pour cela;

- ne pas reprendre les coefficients multiplicateurs d'études analogues pour d'autres zones pour les appliquer à l'impact de $\mathrm{x}$ dans la zone d'étude retenue. Le respect de cette règle de rigueur est nécessaire car les combinaisons des interrelations entre entreprises locales sont en effet le plus souvent structurées différemment d'une zone à l'autre de sorte que liens et fuites seront différents. De même, peuvent différer structurellement la consommation des ménages, les dépenses publiques locales, les achats entre branches non locaux ou les ventes à l'extérieur de la zone. Pour les mêmes raisons, il n'est pas raisonnable de faire une déclaration générale du type « Les festivals musicaux ont un coefficient multiplicateur de revenu de 0,7». En toute rigueur, chaque situation, chaque activité culturelle pour chaque zone possible, devrait être analysée et estimée indépendamment.

\section{Exclure les dépenses des résidents locaux}

Les résultats d'études d'impact sont parfois invoqués pour motiver la demande de soutien public. Le fondement économique est le suivant: le soutien public local de $\mathrm{x}$, obtenu au moyen de recettes fiscales locales, peut constituer un levier optimal permettant d'attirer et/ou d'augmenter la demande (exogène ou autonome) des visiteurs culturels non locaux en biens et services locaux ainsi qu'en facteurs de production locaux, ce qui entraîne l'effet multiplicateur décrit plus haut et ainsi une activité économique locale additionnelle qui n'aurait pas existé sans $\mathrm{x}^{9}$.

Ce fondement précise que seules les dépenses des visiteurs non locaux, dont le motif premier de visite dans la zone est l'existence de $\mathrm{x}$, doivent être incluses dans l'estimation de son

8. Les coefficients estimés pour l'emploi local doivent néanmoins être interprétés avec prudence pour plusieurs raisons : distinction des emplois à temps plein et à temps partiel, recours aux heures supplémentaires sans création nette d'emplois locaux, recours à une main-d'œuvre additionnelle non locale. On ne développera pas davantage cet avertissement pour ne pas alourdir le texte.

9. Le modèle économique implicite à ce raisonnement repose sur plusieurs hypothèses non négligeables : prix en production et des facteurs de production constants, ressources en facteurs capital et travail locaux non pleinement exploitées (capacités de production inemployées ou excédentaires), hausses exogènes de la demande (dépenses dites autonomes). Ces hypothèses seront ici ignorées par souci de simplicité et supposées satisfaites. impact économique. Ce principe peut poser problème pour qui compte invoquer les résultats positifs d'une étude pour justifier le montant des dépenses publiques locales qui est ou pourrait être attribué à $x$. Ôter les dépenses des résidents locaux peut en effet réduire substantiellement l'impact estimé.

Ces dépenses ne contribuent pas à l'impact de x car elles représentent une remise en circulation de sommes qui existaient déjà dans la zone de l'étude d'impact. Il est en effet vraisemblable que la grande majorité des dépenses des résidents locaux qu'on peut associer à x se substitue à des dépenses en biens et services locaux qui auraient eu lieu de toute façon dans la zone. Trente euros dépensés par une famille locale pour un spectacle festivalier local correspondent vraisemblablement à trente euros en moins dépensés au musée, au cinéma, au stade ou au parc d'attractions local. Il y a ainsi simple ré-allocation de dépenses locales dans l'économie locale plutôt qu'une hausse nette réelle de son activité économique. N'offrant aucun stimulus économique net, ces dépenses non contributives ne doivent pas être incluses dans les calculs.

L'application de la règle d'exclusion des dépenses des résidents locaux constitue une mesure de prudence qui permet d'éviter des analyses qui comptabiliseraient toutes les dépenses associées à $\mathrm{x}$, qu'elles soient locales ou non, et qui favoriseraient des conclusions du type : x participe à hauteur de $y$ à l'«activité économique locale», à l'«impact économique brut », au «poids » ou à la « taille économique » de l'économie locale. Le non-respect de cette règle conduit à des surestimations dépourvues de sens justificatif, qui induisent en erreur et ne correspondent pas à une estimation de l'impact économique de x. En ne reposant pas sur l'exclusion des dépenses locales substitutives, ces chiffres ne fournissent aucun élément permettant de savoir si la subvention publique locale accordée à x produi(rai)t ou pas un supplément de revenus et d'emplois, ou si sa suppression produirait ou pas une réduction de l'activité économique locale.

S'il existait des éléments empiriques confortant la possibilité que x maintienne certains résidents locaux dans la zone qui sinon l'auraient quittée pour un voyage, alors leurs dépenses locales pourraient légitimement être considérées comme contribuant à l'impact de x puisque des sommes ont été retenues et dépensées dans la zone plutôt qu'ailleurs. De tels éléments empiriques sont cependant très difficiles à collecter et sont probablement faibles. La convention retenue par les économistes est donc de ne pas tenir compte des dépenses des résidents locaux qu'on peut associer à $\mathrm{x}$, ce qui implique la possibilité que l'estimation de son impact économique soit relativement modeste.

\section{Exclure les dépenses des « changeurs de calendrier» et des visiteurs occasionnels}

Une autre règle à suivre pour estimer correctement l'impact de $\mathrm{x}$ consiste à retirer de l'ensemble des dépenses non locales associées à x celles des « changeurs de calendrier» et celles des visiteurs occasionnels. Qui sont ces individus ? Les premiers sont, dans le cas où $\mathrm{x}$ est par exemple un événement culturel, des visiteurs non locaux qui avaient prévu de visiter la zone où a lieu $\mathrm{x}$ et ont modifié leur calendrier de manière à faire coïncider leurs dates de visite avec celles de x. Les 
seconds sont des visiteurs non locaux déjà présents sur place, attirés par d'autres caractéristiques locales, et qui ont choisi d'être visiteurs de x plutôt que de faire autre chose dans la zone.

Dans les deux cas, les dépenses de restauration, hébergement et commerce de détail ne peuvent pas être attribuées à $\mathrm{x}$ puisqu'elles auraient eu lieu sans $\mathrm{x}$, bien qu'à un autre moment dans l'année pour les changeurs de calendrier. On doit en outre exclure les dépenses de billetterie car elles peuvent être considérées comme des dépenses de substitution: si les visiteurs n'avaient pas payé l'accès à $\mathrm{x}$, ils seraient allés ailleurs dans la zone et auraient dépensé la somme en question. Bien sûr, si des visiteurs non locaux se qualifiant dans leurs réponses au questionnaire de l'étude comme membres de ces deux groupes restent dans la zone plus longtemps qu'ils ne l'auraient fait sans $\mathrm{x}$, il conviendra alors d'inclure leurs dépenses pour les jours supplémentaires dans l'estimation de l'impact de $\mathrm{x}^{10}$.

L'exclusion des dépenses des changeurs de calendrier et des visiteurs occasionnels vient réduire nécessairement l'estimation.

\section{Tenir compte des coûts}

Toute étude d'impact économique doit en principe chercher à tenir compte aussi bien des effets positifs que des effets négatifs que l'on peut associer directement à $\mathrm{x}$, pour disposer d'une évaluation pondérée. Il est certain que l'existence d'un équipement culturel ou la tenue d'un événement culturel engendre localement à court terme toutes sortes de coûts monétaires et non monétaires qu'il est souhaitable, dans la mesure du possible, d'identifier et d'opposer aux bénéfices, de manière à estimer convenablement l'impact. Les parties prenantes locales sont en outre sans doute plus concernées par les bénéfices nets que par les bénéfices bruts que l'on peut associer directement à $\mathrm{x}^{11}$.

Un principe complémentaire consiste ainsi à vérifier avant toute utilisation des résultats d'une étude que les coûts n'ont pas été minorés voire ignorés. Ne pas prêter attention aux coûts conduit en effet à une surestimation incorrecte de l'impact; les mettre en balance avec les bénéfices viendra le réduire et pourra aussi l'annuler voire le rendre négatif, si les coûts excèdent les bénéfices.

Tenir compte des coûts dans l'analyse d'impact transforme celle-ci en analyse bénéfices-coûts, une méthode permettant d'identifier l'ampleur de retours sur investissements. Ce type d'analyse apparaît comme le plus recommandable pour informer le contribuable et aider le décideur, en dépit des difficultés à mesurer correctement les coûts et à les opposer aux bénéfices.

10. Dans le cas par exemple d'un événement culturel, un questionnaire simple inclut habituellement des questions sur la raison de la présence (spectateur, artiste, journaliste, vendeur...), sur le code postal du domicile de la personne interrogée, sur le nombre de jours de présence dans les lieux, sur le nombre de personnes du groupe dont elle fait partie (en famille, avec des amis...), sur les dépenses estimées de ce groupe par type de dépenses (billets, alimentation, commerce de détail, hébergement...) dans et hors la zone d'étude ainsi que des questions permettant d'identifier les changeurs de calendrier et les visiteurs occasionnels.

11. D'où l'utilisation plus haut de l'adjectif «nette » au moment de définir précisément l'objet d'une étude d'impact.
Parmi ces coûts, on peut distinguer des coûts monétaires tels que, par exemple, les investissements dans les équipements locaux, les abattements fiscaux, les subventions locales aux organisations et équipements ainsi que les dispositifs de sécurité publique (police, gendarmerie, pompiers) qu' on peut directement lier à x. Peuvent s'y ajouter des coûts en termes d'encombrement de voies, d'accidents de la route, de vandalisme, de dégradation environnementale, d'hygiène publique, etc.

Une autre catégorie de coûts est ce qu'on appelle les coûts de déplacement ${ }^{12}$. Dans le cas d'un événement culturel par exemple, il est possible que des visiteurs non locaux attirés par l'événement déplacent, évincent ou encore se substituent à d'autres visiteurs qui sinon seraient venus dans la zone mais ne l'ont pas fait, soit parce qu'ils n'ont pas pu trouver d'hébergement, soit parce qu'ils ne sont pas disposés à se mêler à la foule attirée par l'événement. Les données des études d'impact sont tirées d'enquêtes auprès des visiteurs et chaque visiteur non local est considéré a priori comme source de nouvel impact. Le problème est que, si chaque visiteur non local remplace simplement un autre visiteur qui n'est pas venu dans la zone en raison de l'encombrement dû à l'événement, il n'y a alors aucun nouvel impact économique.

Une autre forme de déplacement peut aussi apparaitre lorsqu'un ancien équipement culturel est remplacé par un nouveau et qu'une étude attribue tous les gains économiques locaux à ce dernier. Il est possible qu'en fait la plupart de ces gains puissent déjà être attribués à l'ancien équipement. Seuls les gains marginaux attribuables uniquement au nouvel équipement constituent alors une nouvelle source de revenus individuels et d'emplois locaux. L'autre part des gains est simplement un impact déplacé tiré de l'ancien équipement.

Pour conclure, provisoirement, sur la question de la prise en compte des coûts dans l'évaluation de l'impact économique de $\mathrm{x}$, si certains de ces différents coûts ne peuvent pas être traduits en valeur pécuniaire dans l'analyse et/ou mis en balance avec les bénéfices, ils doivent au moins être décrits précisément, évalués qualitativement et inclus dans une présentation de l'évaluation.

\section{LES RISQUES D'UNE UTILISATION JUSTIFICATIVE DES RÉSULTATS D'IMPACT ÉCONOMIQUE}

Utiliser les résultats de l'analyse d'impact économique pour motiver économiquement le soutien public local à des activités culturelles peut présenter plusieurs inconvénients touchant les objectifs visés par l'utilisateur ainsi que le possible caractère exceptionnel et l'importance relative de ces activités.

12. On parle aussi de «contrainte d'offre» ou de «contrainte de capacité ». En toute rigueur, une des hypothèses du modèle économique implicite de la note 9 est qu'une telle contrainte n'existe pas, ce qui dispenserait d'en parler. Il n'est néanmoins pas inutile de signaler son existence potentielle. 


\section{Des objectifs pécuniaires non neutres culturellement}

Il importe d'avoir conscience que l'analyse d'impact, lorsqu'elle est utilisée pour justifier le soutien public à $\mathrm{x}$, conduit à joindre la politique de création de richesses à la politique culturelle. Le fait d'invoquer des chiffres positifs d'impact amène en effet le décideur public local à intervenir dans le domaine culturel pour atteindre des objectifs uniquement pécuniaires - en matière de revenus, de salaires tirés d'emplois, de recettes fiscales - en utilisant les arts et la culture comme accessoire, en les instrumentalisant. Or, le fait d'atteindre ces objectifs n'est pas une garantie a priori que des objectifs, disons, artistiques et culturels (non pécuniaires) peuvent être atteints.

Pour compléter la mise en garde sur ce risque d'incompatibilité, notons que, face à deux études d'impact de deux projets culturels différents, le décideur sera incité à privilégier celui qui assure le plus gros impact pour un même montant de subvention publique locale. Or rien ne garantit au premier abord que ce choix sera le plus satisfaisant au regard de critères artistiques et culturels.

Enfin, la volonté, disons stratégique, d'attirer des dépenses non locales en vue d'un gain financier net pour une économie locale donnée plutôt que pour (au détriment d') une autre économie locale pourrait conduire à une forme protectionniste de «surenchère » qui pourrait aboutir à une « guerre » de l'événement culturel. Le résultat peut ne pas être neutre culturellement et même conduire à l'excès.

\section{Une « exception culturelle » affaiblie}

À ce risque d'incompatibilité s' ajoute un deuxième risque, celui d'affaiblir l'argument de l' « exception culturelle », argument étendu parfois au-delà des seuls secteurs audiovisuel et cinématographique. Utiliser l'analyse d'impact pour motiver le soutien public à $\mathrm{x}$, en faisant de la politique culturelle une politique utilitaire, signifie qu' on se soucie principalement des revenus et des salaires tirés d'emplois, des sommes qui sont directement et indirectement attribuables à l'existence de x. Le choix de retenir cette analyse affaiblit l'argument de l'« exception culturelle », ceux qui l'invoquent considérant que les biens et services culturels ne sont pas des produits comme les autres car non réductibles à leur seule dimension marchande.

De plus, le fait d'avancer des chiffres d'impact amène à instrumentaliser les services culturels (festivals, spectacles de rue, monuments, sites patrimoniaux, musées, expositions temporaires...) et à en faire des services comme les autres, c'està-dire des services qui produisent de façon ordinaire des revenus et des emplois. Cela peut venir affaiblir leur possible caractère exceptionnel.

\section{L'erreur d'interprétation politique}

Il existe enfin un troisième risque d'utilisation de l'analyse d'impact pour motiver la subvention publique locale à une activité culturelle. Ce risque est lié à une dernière forme de coûts qu'il convient de ne pas ignorer pour estimer correctement l'impact économique local pondéré de $\mathrm{x}$.
Supposons un membre d'un groupe de pression qui dispose d'une évaluation de l'impact de x pour une certaine zone et qui l'invoque pour motiver sa demande de soutien public. Supposons que l'évaluation ait scrupuleusement évité toutes les erreurs de mesure possibles, qu'elle ait réussi passablement à mettre en balance les bénéfices identifiés de x avec ses coûts, et que l'impact net estimé soit (toujours) positif. Un dernier coût reste à considérer pour clore l'analyse. Il s'agit du « coût d'opportunité » des fonds publics locaux dépensés pour soutenir x, c'est-à-dire ce qui est abandonné pour financer sur ces fonds ce dernier. La justification d'un investissement public local suppose en effet que $\mathrm{x}$ doit fournir aux résidents locaux un rendement au moins égal à ce qui serait obtenu pour d'autres projets dans lesquels les autorités locales pourraient investir. Dans ce cas, le coût d'opportunité est la valeur de la meilleure solution de replacement non choisie lorsque la décision de dépenser des fonds publics est prise. Toute étude d'impact sérieuse doit donc identifier cette solution, la mettre en balance avec son objet d'étude et démontrer que c'est bien ce dernier qui est le plus efficace.

L'identification rigoureuse de la meilleure solution de remplacement pourrait cependant impliquer qu'il s'avère préférable, étant donnée la logique de l'analyse, de subventionner sur fonds publics locaux un équipement sportif (un terrain de golf, par exemple), un parc d'attractions, un casino ou un palais des congrès (qui attire avantageusement de nombreux visiteurs non locaux) plutôt que d'aider $\mathrm{x}$. Le risque pour notre lobbyiste culturel est que $\mathrm{x}$ ne soit pas, après évaluation solide, le moyen de produire le plus gros supplément d'activité économique locale, et que son impact net complet s'avère ainsi négatif. L'utilisation justificative de l'argument de l'impact économique peut ainsi s'avérer dangereusement contre-productive pour les arts et la culture. C'est d'ailleurs sans doute pour cette raison que les études publiées ne cherchent habituellement pas à évaluer le coût d'opportunité des fonds publics utilisés pour soutenir leur objet d'étude. Bien sûr, rien n'exclut a priori que x permette de produire l'impact le plus important ; il convient pourtant d'en faire la démonstration incontestable, ce qui au premier abord n'est pas évident ${ }^{13}$.

En d'autres termes, il ne faut pas commettre l'erreur d'interprétation politique, c'est-à-dire supposer incorrectement qu'un impact positif est une condition suffisante pour prétendre à l'aide publique sans considérer les coûts d'opportunité de détournement d'une telle aide vers d'autres investissements publics potentiellement plus rentables. Prendre au sérieux l'analyse d'impact pour justifier le soutien public local implique la mise en concurrence de l'activité culturelle locale étudiée (celle de $\mathrm{x}$ ) avec n'importe quelle autre activité industrielle ou commerciale locale susceptible d'être soutenue, à moins de faire le jugement de valeur contestable selon lequel créer des revenus et des emplois locaux grâce au levier culturel local étudié importe plus que d'en créer grâce à tout autre levier local. Comme nous l'avons dit, cette mise en concurrence, qui néglige l'éventuelle nature exceptionnelle de services culturels, peut s'avérer, après évaluation solide, défavorable pour qui souhaite voir $\mathrm{x}$ bénéficier d'un soutien public.

13. La question d'une démonstration en analyse dynamique sera ignorée ici, les choses pouvant bien entendu se modifier dans le temps. 
Autrement dit encore, si l'analyse d'impact est prise au sérieux pour justifier la subvention publique, alors quiconque commande une étude à visée justificative doit accepter préalablement le fait que celle-ci puisse démontrer qu'il est préférable pour les contribuables locaux de supprimer ou de ne pas accorder à $\mathrm{x}$ tel soutien public local mais de le verser plutôt à une autre activité, culturelle ou pas. Si, après estimation, il en est ainsi mais que cette conclusion est niée et la subvention maintenue ou accordée à $x$, c'est que l'analyse d'impact n'a pas été prise au sérieux par le commettant de l'étude ; il en attendait sans doute des résultats particuliers, par exemple pour légitimer a posteriori une subvention publique décidée sur d'autres motifs et qui, de toute façon, n'aurait pas été remise en cause. Dans ce cas, un argument(aire) non économique sera avancé en guise de justification et l'étude d'impact n'aura donc servi à rien, la commande n'ayant pas été passée honnêtement. Par surcroît, des ressources en temps, en énergie et en fonds publics dépensées pour l'étude auront été gaspillées.

\section{POUR CONCLURE}

Au terme de ce rappel des premiers principes de l'analyse d'impact économique local d'une activité culturelle, que conclure ? Cette analyse a une valeur descriptive et informative indéniable puisqu'elle permet notamment d'améliorer la connaissance des interdépendances des différents secteurs d'activité locaux bénéficiant directement et indirectement des dépenses liées à l'activité culturelle étudiée. De plus, les résultats d'une étude d'impact correctement faite peuvent être invoqués pour motiver une demande de soutien public local.

L'utilisation justificative doit toutefois être faite avec circonspection. En particulier, le fait d'allier politique culturelle et politique de création de richesses est risqué pour trois raisons : cette alliance peut s'avérer non neutre culturellement, affaiblir l'argument de l' «exception culturelle» et mettre en concurrence, dans la lutte pour les ressources publiques rares, une activité culturelle locale avec n'importe quelle autre activité industrielle ou commerciale locale susceptible d'être soutenue. Ces trois périls résultent du choix de retenir l'analyse d'impact pour justifier le soutien public local. Face à eux, la prudence est clairement de mise.

\section{Aide-mémoire des conditions nécessaires pour une étude d'impact économique local d'une activité culturelle}

Avant de diffuser les résultats d'une étude d'impact économique local d'une activité culturelle, il convient de s'assurer que les conditions cidessous sont réunies:

- les dépenses des résidents locaux ont été exclues;

- les dépenses des changeurs de calendrier et/ou des visiteurs occasionnels ont été exclues;

- les estimations des coefficients multiplicateurs ont été calculées à l'aide d'un modèle entrées/sorties de l'économie locale (et non à partir des résultats d'études analogues);

- les estimations des coefficients multiplicateurs portent sur les revenus des résidents locaux plutôt que sur les ventes des entreprises locales;

-les coûts de l'objet d'étude ont été pris en considération, dont son coût d'opportunité.

\section{RÉSUMÉ}

L'impact économique local d'un équipement ou d'un événement culturel est attribuable aux dépenses de ceux qui les fréquentent. Il ne peut être solidement estimé qu'en respectant, une fois compris ce qu'il mesure, plusieurs premières règles de méthode : utilisation d'un modèle économique local $a d$ hoc, exclusion de plusieurs types de dépenses, prise en considération de ses coûts. Cette estimation faite, les résultats ne peuvent toutefois être utilisés qu'avec prudence pour motiver la subvention publique locale à une activité culturelle. Cette note détaille les raisons justifiant cette attitude.

\section{ABSTRACT}

The local economic impact of a cultural event or facility derives from the money spent by its audience. It can be reliably measured - once it is understood what exactly is being measured - only by observing several methodological ground rules: use of an ad hoc local economic model; exclusion of certain kinds of expenditure; calculation of costs. After estimation, the findings require that caution be exercised when using them for justifying local government subsidies for a cultural activity. This note sets out the reasons underlying this attitude. 